\title{
Effective locality and gauge invariance
}

\author{
T. Grandou, ${ }^{1, a}$ "On behalf of a collaboration with H.M. Fried and R. Hofmann" \\ ${ }^{1}$ Institut de Physique de Nice, UMR-CNRS 7010, Valbonne 06560, France,
}

\begin{abstract}
A few years ago, based on standard functional manipulations, an unexpected property was proven to be satisfied by the fermionic Green's functions of QCD, and dubbed effective locality. This feature of QCD is non-perturbative as it results from a full integration of the fermionic and gluonic degrees of freedom. Previous derivations of effective locality are reviewed, corrected, and enlarged. Focussing on the way nonabelian gauge invariance is realized in the non-perturbative regime of QCD, the meaning of effective locality is proposed.
\end{abstract}

\section{Introduction}

In a series of recent articles [1-5] a property of the non-perturbative fermionic Green's functions of QCD has been put forward under the name effective locality, and at face value this property can be summarized as follows.

For any fermionic 2n-point Green's functions and related amplitudes, the full gauge-fixed sum of linear, cubic and quartic gluonic interactions, fermionic loops included, results in a local contacttype interaction. This local interaction is mediated by a tensorial field which is antisymmetric both in Lorentz and color indices. Moreover, the resulting sum appears to be fully gauge-fixing independent, that is, gauge-invariant.

This result is a non-expected one because integrations of elementary degrees of freedom ordinarily result in highly non-local structures. The 'effective locality' denomination accounts for this rather unusual circumstance. In the pure euclidean Yang Mills case, though, and up to the first non-trivial orders of a semi-classical expansion, effective locality was observed a welcome property in an attempt to construct a formulation dual to the original Yang Mills theory [6].

Now, apart from a supersymmetric extension, QCD is not known to possess any dual formulation, and the full effective locality functional expressions certainly attest to this difficulty. However, like in the pure Yang Mills situation of [6], effective locality offers a useful means to learn about nonperturbative physics in QCD, and this based on first principles and standard functional operations.

To begin with, it is worth reviewing the constructions of the Green's functions generating functionals of QED and QCD, and to contrast them at one essential point. The statement of effective locality can then be exposed in the simpler situation where it was first discovered [1], before being extended to the full non approximated theory [2]. In these two previous cases, made was used of socalled Schwinger/Fradkin's representations for a quark propagating in a background gluonic field. As it turns out, though, effective locality holds true irrespective of Schwinger/Fradkin's representations, and this helps to disclose one of the deepest aspect of this newly discovered property [7].

${ }^{\mathrm{a}}$ e-mail: thierry.grandou@inphyni.cnrs.fr 


\section{QED and QCD generating functionals}

A long known aspect of QED quantization is that manifest covariance and manifest gauge invariance are competing aspects of a generating functional (GF) construction. Quickly reviewing the conventional Schwinger solution for the generating functionals of QED and QCD, expressions are obtained which, beyond appearances, differ on an essential point.

\subsection{QED generating functional}

For QED, the free photonic Lagrangian density reads,

$$
\mathcal{L}_{0}=-\frac{1}{4} f^{\mu v} f_{\mu v}=-\frac{1}{4}\left(\partial^{\mu} A^{v}-\partial^{v} A^{\mu}\right)\left(\partial_{\mu} A_{v}-\partial_{v} A_{\mu}\right)
$$

The corresponding action may be written as,

$$
\int \mathrm{d}^{4} x \mathcal{L}_{0}=-\frac{1}{2} \int A^{\mu}\left(-\partial^{2}\right) A_{\mu}+\frac{\lambda}{2} \int\left(\partial^{\mu} A_{\mu}\right)^{2},
$$

at the value of $\lambda=1$. In the right hand side of (2), this second 'unconvenient term', as E. Fermi called it, can be used to generate any choice of a covariant gauge by treating this term as an interaction term. Subsequent enforcing of charge conservation, then, allows one to recover gauge invariance. This recovering is therfeore an indirect two-step procedure, a price to pay in order to preserve covariance from the onset. For the QED generating functional one starts from the free field piece $(e=0, \lambda=0)$ : With $D_{F \mu \nu}^{(0)}=g_{\mu \nu} D_{F},\left(-\partial^{2}\right) D_{F}=\delta^{4}$, a new free-field generating functional is obtained,

$$
\begin{aligned}
Z_{0}^{(\zeta)}\{j\} & =\left.e^{\frac{i}{2} \lambda \int\left(\partial^{\mu} A_{\mu}\right)^{2}}\right|_{A \rightarrow \frac{1}{i} \frac{\delta}{\delta j}} \cdot e^{\frac{i}{2} \int j \cdot D_{\mathrm{F}}^{(0)} \cdot j} \\
& =e^{-\frac{i}{2} \operatorname{Tr} \ln \left[1-\lambda\left(\partial \otimes \partial / \partial^{2}\right)\right]} e^{\frac{i}{2} \int j \cdot D_{\mathrm{F}}^{(\zeta)} \cdot j}
\end{aligned}
$$

where,

$$
D_{\mathrm{F} \mu \nu}^{(\zeta)}=\left[g_{\mu \nu}-\zeta \partial_{\mu} \partial_{v} / \partial^{2}\right] D_{\mathrm{F}}, \quad \zeta=\frac{\lambda}{1-\lambda} .
$$

Note that the Tr-Log term of (3) is an infinite phase factor representing the sum of the vacuum energies generated by longitudinal and time-like photons, with a weight $\lambda$ arbitrarily inserted. This quantity can be removed by an appropriate version of normal ordering, or more simply, be absorbed into an overall normalization constant.

Fermionic fields can be integrated out in the presence of fermionic sources $\bar{\eta}$ and $\eta$. This operation yields the standard gluonic field fiunctionals,

$$
G_{F}[A]=[\gamma \cdot i(\partial-i e A)-m]^{-1}, \quad L[A]=\operatorname{Tr} \ln \left[1-i g \gamma \cdot A S_{\mathrm{F}}\right], \quad S_{\mathrm{F}}=G_{\mathrm{F}}[0],
$$

in terms of which the full GF of QED is obtained by letting these functionals operate on the free field GF,

$$
Z_{\mathrm{QED}}^{(\zeta)}[j, \eta, \bar{\eta}]=\left.\mathcal{N} e^{i \int \bar{\eta} \cdot G_{\mathrm{F}}[A] \cdot \eta+L[A]+\frac{i}{2} \lambda \int\left(\partial^{\mu} A_{\mu}\right)^{2}}\right|_{A \rightarrow \frac{1}{i} \frac{\delta}{\delta j}} \cdot e^{\frac{i}{2} \int j \cdot D_{\mathrm{F}}^{(0)} \cdot j} .
$$

Now for any polynomial and exponential functional $\mathcal{F}[A]$, the following differential identity holds,

$$
\mathcal{F}\left[\frac{1}{i} \frac{\delta}{\delta j}\right] \cdot e^{\frac{i}{2} \int j \cdot D_{\mathrm{F}}^{(\zeta)} \cdot j}=\left.e^{\frac{i}{2} \int j \cdot D_{\mathrm{F}}^{(\zeta)} \cdot j} \cdot e^{\mathfrak{D}_{A}} \cdot \mathcal{F}[A]\right|_{A=\int D_{\mathrm{F}}^{(\zeta)} \cdot j}
$$


where $\mathfrak{D}_{A}^{(\zeta)}$, is the so-called linkage operator, $\mathfrak{D}_{A}^{(\zeta)}=-\frac{i}{2} \int \mathrm{d}^{4} x \int \mathrm{d}^{4} y \frac{\delta}{\delta A(x)} \cdot D_{\mathrm{F}}^{(\zeta)}(x-y) \cdot \frac{\delta}{\delta A(y)}$. This identity can be used to eventually write,

$$
Z_{\mathrm{QED}}^{(\zeta)}[j, \eta, \bar{\eta}]=\left.\mathcal{N} e^{\frac{i}{2} \int j \cdot D_{\mathrm{F}}^{(\zeta)} \cdot j} \cdot e^{\mathfrak{D}_{A}^{(\zeta)}} \cdot e^{i \int \bar{\eta} \cdot G_{\mathrm{F}}[A] \cdot \eta+L[A]}\right|_{A=\int D_{\mathrm{F}}^{(\zeta)} \cdot j} \cdot
$$

This is nothing but the formal solution for the QED generating functional which would result from a linear and covariant gauge fixing term $\frac{-1}{2(1-\lambda)}(\partial \cdot A)^{2}$. Accordingly, the Green's functions given by (8) cannot display gauge invariance: The bare photon propagator is explicitly gauge-dependent and it is only through the control of Ward identities that radiative corrections invoking this propagator are proven to be gauge invariant. Likewise, using such a gauge dependent photon propagator does not prevent properly defined $S$ - matrix elements from being gauge-invariant, by virtue of the LSZ reduction formula and equivalence theorem [8].

For our current purpose, the point to be retained here is that recovering gauge invariance goes along some circuitous procedure.

\subsection{QCD generating functional}

The standard functional steps of Schwinger's construction can be taken here again, out of a Lagrangian density of $\mathcal{L}_{\mathrm{QCD}}=-\frac{1}{4} F_{\mu \nu}^{a} F^{\mu \nu a}-\bar{\psi} \cdot\left[\gamma^{\mu} i\left(\partial_{\mu}-i g A_{\mu}^{a} \lambda^{a}\right)-m\right] \cdot \psi$, where $F_{\mu \nu}^{a}=\partial_{\mu} A_{\nu}^{a}-\partial_{\nu} A_{\mu}^{a}+g f^{a b c} A_{\mu}^{b} A_{\nu}^{c}$, and the numbers $f^{a b c}$ are the customary structure constants of the $S U_{c}(3)$ Lie algebra.

One writes,

$$
Z_{Q C D}^{(?)}[j, \bar{\eta}, \eta]=\mathcal{N} e^{i \int \mathcal{L}_{Q C D}^{\prime}\left[\frac{1}{i} \frac{\delta}{\delta j}\right]} e^{\frac{i}{2} \int j \cdot D_{\mathrm{F}}^{(?)} \cdot j}
$$

with the Lagrangian interaction part,

$$
\mathcal{L}^{\prime}{ }_{\mathrm{QCD}}[A]=\mathcal{L}^{\prime}[A]+\bar{\eta} G_{\mathrm{F}}[A] \eta+L[A] .
$$

and where, exactly like in QED, the following propagator doesn't exist,

$$
\left(D_{\mathrm{F}}^{(?)^{-1}}\right)_{\mu \nu}^{a b}=-i \delta^{a b}\left[g_{\mu \nu} \partial^{2}-\partial_{\mu} \partial_{\nu}\right]
$$

Now in QCD, unlike QED, one can establish the exact relation,

$$
\int \mathcal{L}^{\prime}[A]=-\frac{1}{4} \int F^{2}+\frac{1}{2} \int A_{a}^{\mu}\left(-\partial^{2}\right) A_{\mu}^{a}-\frac{1}{2} \int\left(\partial^{\mu} A_{\mu}^{a}\right)^{2},
$$

and emphasize that in QED this term is zero. Using this relation the $\partial_{\mu} \partial_{v}$ piece of (11) is cancelled so as to transform (11) into the well defined and covariant Feynman propagator $D_{F}^{(0)}$. Omitting the fermionic part for a while, one thus writes,

$$
Z^{(0)}[j]=\left.\mathcal{N} e^{-\frac{i}{4} \int F^{2}+\frac{i}{2} \int A_{\mu}^{a}\left(-\partial^{2}\right) A_{a}^{\mu}}\right|_{A \rightarrow \frac{1}{i} \frac{\delta}{\delta j}} e^{\frac{i}{2} \int j \cdot D_{\mathrm{F}}^{(0)} \cdot j},
$$

Had the same ' $\lambda$-game' as in QED been played here also, then one discovered that the value of $\lambda=1$ was a possible choice. In other words, that both manifest gauge invariance and Lorentz covariance could be maintained on the same time, contrarily to the case of QED. This however seems to privilege $D_{F}^{(0)}$, the Feynman propagator. Now, that it isn't really so is disclosed by the simple trick of adding 
and subtracting out a given linear covariant gauge fixing density of $\frac{1}{2 \zeta}(\partial \cdot A)^{2}$, passing from $D_{F}^{(?)}$ to the invertible $D_{F}^{(\zeta)}$,

$$
\left(D_{\mathrm{F}}^{(\zeta)^{-1}}\right)_{\mu \nu}^{a b}=-i \delta^{a b}\left[g_{\mu \nu} \partial^{2}+\left(\frac{1}{\zeta}-1\right) \partial_{\mu} \partial_{\nu}\right],
$$

which at $\zeta=1$ comprises the Feynman case of $D_{F}^{(0)}$. Restoring the fermionic dependences and using identity (7) again, one obtains,

$$
\begin{aligned}
Z_{\mathrm{QCD}}[j, \bar{\eta}, \eta] & =\mathcal{N} e^{\frac{i}{2} \int j \cdot D_{\mathrm{F}}^{(\zeta)} \cdot j} \\
& \times\left. e^{-\frac{i}{2} \int \frac{\delta}{\delta A} \cdot D_{\mathrm{F}}^{(\zeta)} \cdot \frac{\delta}{\delta A}} \cdot e^{-\frac{i}{4} \int F^{2}+\frac{i}{2} \int A \cdot\left(D_{\mathrm{F}}^{(\zeta)}\right)^{-1} \cdot A} \cdot e^{i \int \bar{\eta} \cdot G[G] \cdot \eta+L[A]}\right|_{A=\int D_{\mathrm{F}}^{(\zeta)} \cdot j} .
\end{aligned}
$$

The same operations can be undertaken with an axial planar gauge fixing density of $\frac{1}{2 \zeta}(\mathbf{n} \cdot A)^{2}$, where $\mathbf{n}$ is a lightlike vector, $\mathbf{n}^{2}=0$. Then, in the limit of $\zeta \rightarrow 0$, one gets the axial planar gauge field function, propagating the 2 gauge field physical degrees of freedom only,

$$
\left(D_{\mathrm{F}}^{(\mathbf{n})}\right)_{\mu \nu}^{a b}(K)=-i \delta^{a b}\left(\frac{g_{\mu \nu}}{K^{2}+i \varepsilon}-\frac{K_{\mu} n_{v}+K_{v} n_{\mu}}{\left(K^{2}+i \varepsilon\right)(K \cdot \mathbf{n})}\right), \operatorname{Tr}\left[g_{\mu \nu}-\frac{K_{\mu} n_{v}+K_{v} n_{\mu}}{K \cdot \mathbf{n}}\right]=2,
$$

while the associated generating functional reads,

$$
\begin{aligned}
Z_{\mathrm{QCD}}[j, \bar{\eta}, \eta] & =\mathcal{N} e^{\frac{i}{2} \int j \cdot D_{\mathrm{F}}^{(\mathbf{n})} \cdot j} \\
& \times\left. e^{-\frac{i}{2} \int \frac{\delta}{\delta A} \cdot D_{\mathrm{F}}^{(\mathbf{n})} \cdot \frac{\delta}{\delta A}} \cdot e^{-\frac{i}{4} \int F^{2}+\frac{i}{2} \int A \cdot\left(D_{\mathrm{F}}^{(\mathbf{n})}\right)^{-1} \cdot A} \cdot e^{i \int \bar{\eta} \cdot G_{\mathrm{F}}[A] \cdot \eta+L[A]}\right|_{A=\int D_{\mathrm{F}}^{(\mathbf{n})} \cdot j} .
\end{aligned}
$$

That is, as many identical GF forms as desired can be obtained in this way, differing only the intermediate gluonic free field functions, $D_{F}^{(0)}, D_{F}^{(\zeta)}, D_{F}^{(n)}$, etc..

Out of these considerations, a first set of points can already be put forth.

- In QED ( $\lambda=1$ cannot be taken) these standard functional operations amount to a quantization in a given covariant gauge. As well known, manifest Lorentz covariance is preserved at the expense of manifest gauge invariance whose recovering is indirect.

- In QCD: Manifest Lorentz covariance and manifest gauge invariance can be preserved at a time. The same ' $\lambda$-game' can be played, with the discovery that in QCD, $\lambda=1$ can be taken.

- However, as disclosed by the trick of adding/subtracting a given gauge fixing density to the Lagrangian, none of the several forms of the QCD $G F \mathrm{~s}$ so obtained, with $D_{\mathrm{F}}^{(n)}, D_{\mathrm{F}}^{(\zeta)}$ or any other propagator do correspond to the usual QCD GFs in the associated gauge.

- This is because no actual gauge fixing has been chosen indeed. There is but one possibility. Gauge field functions (propagators) must show up as necessary intermediates [12] and not affect the fermionic momenta of the QCD GFs ..

And this is what the property of effective locality displays.

Are there provisos to this procedure? There shouldn't be any in principle since adding/subtracting amounts to nothing; now some surprises could come about according to the order operations are carried out, in particular when it comes to integrating over some covariant gauge field configurations. Now, this problem is avoided here, where quantization, whatever the gauge choice, is achieved by means of functional differentiation rather than functional integration. In the end, the fact that final results cannot depend on any form of gluonic free field function turns out to offer an efficient way of controlling calculations. 


\section{Effective locality}

Before being extended to the full non approximated case [2]. the phenomenon of effective locality has been discovered in QCD relying first on the eikonal and quenching approximations [1]. In all cases the following standard 'linearization' is used $[6,9]$,

$$
e^{-\frac{i}{4} \int F^{2}}=\mathcal{N}^{\prime} \int \mathrm{d}[\chi] e^{\frac{i}{4} \int\left(\chi_{\mu \nu}^{a}\right)^{2}+\frac{i}{2} \int \chi_{a}^{\mu \nu} F_{\mu \nu}^{a}}
$$

with an integration measure of,

$$
\int \mathrm{d}[\chi]=\prod_{z} \prod_{a} \prod_{\mu<v} \int \mathrm{d}\left[\chi_{\mu \nu}^{a}\right](z) .
$$

It may be observed that the linearization (18) breaks the manifest gauge invariance of the left hand side. In order to remedy this, one may either gauge the $\chi$-field (H. Reinhardt et al. [6]), or integrate exactly the $\chi$-field dependences; in the 'short version', this is doable with the help of Random Matrix theory $[4,10]$. However, so long as gauge invariance is concerned only, none of the above possibilities is necessary as effective locality brings about a remarkable simplification as it should appear clearly enough in the sequel.

To proceed, any $D_{F}$-dependent form of $Z_{\mathrm{QCD}}[j, \bar{\eta}, \eta]$ can be used, such as

$$
\left.\mathcal{N} \int \mathrm{d}[\chi] e^{\frac{i}{4} \int \chi^{2}} e^{\mathfrak{D}_{A}^{(0)}} e^{-\frac{i}{2} \int \chi \cdot F+\frac{i}{2} \int A \cdot\left(-\partial^{2}\right) \cdot A} \cdot e^{i \int \bar{\eta} \cdot G_{\mathrm{F}}[A] \cdot \eta+L[A]}\right|_{A=\int D_{\mathrm{F}}^{(0)} j}
$$

For the fermionic field functional in a background field configuration $A_{\mu}^{a}$ (See Eq.(5)), Fradkin/Schwinger's representations are used as for example in the 'mixed case',

$$
\begin{aligned}
\left\langle p\left|G_{F}[A]\right| y\right\rangle= & i e^{-i p \cdot y} \int_{0}^{\infty} \mathrm{d} s e^{-i s m^{2}} e^{-\frac{1}{2} \operatorname{Tr} \ln (2 h)} \\
& \times \int \mathrm{d}[u]\{m-i \gamma \cdot[p-g A(y-u(s))]\} e^{\frac{i}{4} \int_{0}^{s} \mathrm{~d} s^{\prime}\left[u^{\prime}\left(s^{\prime}\right)\right]^{2}} e^{i p \cdot u(s)} \\
& \times\left(e^{g \int_{0}^{s} \mathrm{~d} s^{\prime} \sigma \cdot \mathbf{F}\left(y-u\left(s^{\prime}\right)\right)} e^{-i g \int_{0}^{s} \mathrm{~d} s^{\prime} u^{\prime}\left(s^{\prime}\right) \cdot \mathbf{A}\left(y-u\left(s^{\prime}\right)\right)}\right)_{+}
\end{aligned}
$$

with,

$$
h\left(s_{1}, s_{2}\right)=s_{1} \Theta\left(s_{2}-s_{1}\right)+s_{2} \Theta\left(s_{1}-s_{2}\right), \quad h^{-1}\left(s_{1}, s_{2}\right)=\frac{\partial}{\partial s_{1}} \frac{\partial}{\partial s_{2}} \delta\left(s_{1}-s_{2}\right) .
$$

To avoid exponentials of exponentials, functionals $G_{F}[A]$ will be brought 'downstairs' by means of functional differentiations w.r.t the fermionic sources $(\bar{\eta}, \eta)$ and this is why effective locality is most easily read off fermionic Green's functions. The Fradkin field variable is $u(s)$ and the subscript + stands for $s$-Schwinger proper time ordering prescription. Fradkin's representations can hardly be thought of as being overly simple, but they are exact. A similar form exists also for the $A_{\mu}^{a}$-functional $L[A]$ of (5), which is set to 0 as the quenching quenching approximation is used. Completed with the eikonal approximation, one can get the simplest derivation of the effective locality property.

\subsection{Effective locality short version}

Ordered exponentials are fairly complicated objects but within an eikonal treatment such an ordered exponential as that of (21) reduces to (with $u(s)=s p$, the 'straight-line' approximation inherent to 
the eikonal),

$$
\begin{aligned}
\left(e^{i g p^{\mu} \int_{-\infty}^{+\infty} \mathrm{d} s A_{\mu}^{a}(y-s p) T^{a}}\right)_{+} & =\mathcal{N} \int \mathrm{d}[\alpha] \int \mathrm{d}[\Omega] \\
& \times e^{-i \int_{-\infty}^{+\infty} \mathrm{d} s \Omega^{a}(s)\left[\alpha^{a}(s)-g p^{\mu} A_{\mu}^{a}(y-s p)\right]} \\
& \times\left(e^{i \int_{-\infty}^{+\infty} \mathrm{d} s \alpha^{a}(s) T^{a}}\right)_{+}
\end{aligned}
$$

where $\alpha$ and $\Omega$ are extra field variables introduced so as to take the $A_{\mu}^{a}$ - dependences outside of the proper-time ordering. It will matter to integrate them out in an exact way so as to be guaranteed to deal with the original expression. At strong coupling $g>>1$, this will be doable relying on a random matrix calculation [4]. The case of a 4-point Green's function can be taken for the sake of illustration. Up to some relevant normalization, one gets,

$$
\begin{aligned}
& \prod_{i=1}^{2} \int \mathrm{d} s_{i} \int \mathrm{d} u_{i}\left(s_{i}\right) \int \mathrm{d} \alpha_{i}\left(s_{i}\right) \int \mathrm{d} \Omega_{i}\left(s_{i}\right)(\ddots) \\
& \left.\int \mathrm{d}[\chi] e^{\frac{i}{4} \int \chi^{2}} e^{\mathfrak{D}_{A}^{(0)}} e^{+\frac{i}{2} \int A_{\mu}^{a} K_{a b}^{\mu v} A_{v}^{b}} e^{i \int Q_{\mu}^{a} A_{a}^{\mu}}\right|_{A \rightarrow 0}
\end{aligned}
$$

with,

$$
\begin{gathered}
K_{\mu \nu}^{a b}=g f^{a b c} \chi_{\mu \nu}^{c}+\left(D_{\mathrm{F}}^{(0)^{-1}}\right)_{\mu \nu}^{a b}, \quad Q_{\mu}^{a}=-\partial^{v} \chi_{\mu \nu}^{a}+g\left[R_{1, \mu}^{a}+R_{2, \mu}^{a}\right] . \\
R_{i, \mu}^{a}(z)=p_{i, \mu} \int \mathrm{d} s_{i} \Omega_{i}^{a}\left(s_{i}\right) \delta^{4}\left(z-y_{i}+s_{i} p_{i}\right), \quad i=1,2,
\end{gathered}
$$

and the core of effective locality shows up in the following functional operation,

$$
\begin{gathered}
\left.e^{-\frac{i}{2} \int \frac{\delta}{\delta A} \cdot D_{\mathrm{F}}^{(0)} \cdot \frac{\delta}{\delta A}} \cdot e^{+\frac{i}{2} \int A \cdot K \cdot A+i \int A \cdot Q}\right|_{A \rightarrow 0} \\
=e^{-\frac{1}{2} \operatorname{Tr} \ln \left(1-D_{\mathrm{F}}^{(0)} \cdot K\right)} \cdot e^{\frac{i}{2} \int Q \cdot\left[D_{\mathrm{F}}^{(0)} \cdot\left(1-K \cdot D_{\mathrm{F}}^{(0)}\right)^{-1}\right] \cdot Q} .
\end{gathered}
$$

because,

$$
\begin{aligned}
D_{\mathrm{F}}^{(0)} \cdot\left(1-K \cdot D_{\mathrm{F}}^{(0)}\right)^{-1} & =D_{\mathrm{F}}^{(0)} \cdot\left(1-\left[g f \cdot \chi+D_{\mathrm{F}}^{(0)^{-1}}\right] \cdot D_{\mathrm{F}}^{(0)}\right)^{-1} \\
& =-(g f \cdot \chi)^{-1}
\end{aligned}
$$

and,

$$
\left\langle x\left|(g f \cdot \chi)^{-1}\right| y\right\rangle=(g f \cdot \chi)^{-1}(x) \delta^{(4)}(x-y) .
$$

In the end, the 4-point Green's function goes like,

$$
\begin{array}{r}
\mathcal{N} \prod_{i=1}^{2} \int \mathrm{d} s_{i} \int \mathrm{d} u_{i}\left(s_{i}\right) \int \mathrm{d} \alpha_{i}\left(s_{i}\right) \int \mathrm{d} \Omega_{i}\left(s_{i}\right)(\ddots) \int \mathrm{d}[\chi] e^{\frac{i}{4} \int \chi^{2}} \\
\cdot \frac{1}{\sqrt{\operatorname{det}(f \cdot \chi)}} \cdot e^{-\frac{i}{2} \int \mathrm{d}^{4} z Q(z) \cdot(g f \cdot \chi(z))^{-1} \cdot Q(z)}
\end{array}
$$

with,

$$
Q_{\mu}^{a}=-\partial^{v} \chi_{\mu \nu}^{a}+g\left[R_{1, \mu}^{a}+R_{2, \mu}^{a}\right]
$$

This result, 
- is local and gauge-independent,

- cannot happen in the abelian QED case as long hoped (R.P. Feynman, quoted in A. Zee's book),

- generalizes to $2 n$-pt fermionic Green's functions,

- when truncated to the YM case, reproduces the structure found by H. Reinhardt et al. years ago in the euclidean,

- lends itself to (analytically continued) Random Matrix exact calculations.

\subsection{Effective locality full version}

The same example of a 4-point Green's function can be taken. That is,

$$
\begin{aligned}
& M\left(x_{1}, y_{1} ; x_{2}, y_{2}\right) \\
& =\left.\frac{\delta}{\delta \bar{\eta}\left(y_{1}\right)} \cdot \frac{\delta}{\delta \eta\left(x_{1}\right)} \cdot \frac{\delta}{\delta \bar{\eta}\left(y_{2}\right)} \cdot \frac{\delta}{\delta \eta\left(x_{2}\right)} \cdot \mathcal{Z}\{j, \bar{\eta}, \eta\}\right|_{\eta=\bar{\eta}=0 ; j=0} \\
& =\left.\mathcal{N} \int d[\chi] e^{\frac{i}{4} \int \chi^{2}} e^{\mathfrak{D}_{A}^{(\zeta)}} e^{+\frac{i}{2} \int \chi \cdot \mathbf{F}+\frac{i}{2} \int A \cdot\left(\mathbf{D}_{F}^{(S)}\right)^{-1} \cdot A} G_{\mathrm{F}}\left(x_{1}, y_{1} \mid g A\right) G_{\mathrm{F}}\left(x_{2}, y_{2} \mid g A\right) e^{L[A]}\right|_{A=0}
\end{aligned}
$$

But now, gathering all gaussian dependences of $G_{F}(1 \mid A)$ and $G_{F}(2 \mid A)$ in an overall expression like,

$$
\exp \left[\frac{i}{2} \int \mathrm{d}^{4} z A_{a}^{\mu}(z) K_{\mu \nu}^{a b}(z) A_{b}^{v}(z)+i \int \mathrm{d}^{4} z Q_{a}^{\mu}(z) A_{\mu}^{a}(z)\right]
$$

requires that 2 extra auxiliary variables be introduced. With $(i=1,2)$,

$$
\Phi_{i, \mu \nu}^{a}\left(u_{i} ; z\right) \equiv \int_{0}^{s_{i}} \mathrm{~d} s_{i}^{\prime} \delta^{(4)}\left(z-y_{i}+u_{i}\left(s_{i}^{\prime}\right)\right) \Phi_{i, \mu v}^{a}\left(s_{i}^{\prime}\right) .
$$

the 'kernel' $K$ receives an additional spin contribution of,

$$
K_{s p i n}^{a b}(z)=2 g^{2} f^{a b c} \Phi_{1, \mu \nu}^{c}\left(u_{1} ; z\right)+2 g^{2} f^{a b c} \Phi_{2, \mu \nu}^{c}\left(u_{2} ; z\right)
$$

while 'currents' can be identified to

$$
\begin{array}{r}
Q_{\mu}^{a}(z)=-2 g \partial^{v} \Phi_{\mathrm{I}, \nu \mu}^{a}(z)-g \int_{0}^{s_{1}} \mathrm{~d} s_{1}^{\prime} \delta^{(4)}\left(z-y_{1}+u_{1}\left(s_{1}^{\prime}\right)\right) u_{1, \mu}^{\prime}\left(s_{1}^{\prime}\right) \Omega_{1}^{a}\left(s_{1}^{\prime}\right) \\
-2 g \partial^{v} \Phi_{2, \nu \mu}^{a}(z)-g \int_{0}^{s_{2}} \mathrm{~d} s_{2}^{\prime} \delta^{(4)}\left(z-y_{2}+u_{2}\left(s_{2}^{\prime}\right)\right) u_{2, \mu}^{\prime}\left(s_{2}^{\prime}\right) \Omega_{2}^{a}\left(s_{2}^{\prime}\right)
\end{array}
$$

and easily generalized to higher number of points Green's functions in a formally similar way. Neglecting spins and adopting the straight-line approximation of $u_{i}\left(s_{i}^{\prime}\right),(26)$ is recovered as it should. The fermionic determinant being restored (i.e. no quenching), the needed functional operation is now,

$$
\exp \left[-\frac{i}{2} \int \frac{\delta}{\delta A} \cdot D_{\mathrm{F}}^{(0)} \cdot \frac{\delta}{\delta A}\right] \cdot \exp \left[\frac{i}{2} \int A \cdot \bar{K} \cdot A+i \int \bar{Q} \cdot A\right] \cdot \exp (L[A]),
$$

followed by the prescription of cancelling the potentials $A_{\mu}^{a}$, and where,

$$
\begin{aligned}
& \left\langle z\left|\bar{K}_{\mu \nu}^{a b}\right| z^{\prime}\right\rangle=\left[K_{\sin _{\mu \nu} a b}(z)+g f^{a b c} \chi_{\mu \nu}^{c}(z)\right] \delta^{(4)}\left(z-z^{\prime}\right)+\left\langle z\left|\left(D_{F}^{(0)}\right)^{-1}\right|_{\mu \nu}^{a b} \mid z^{\prime}\right\rangle, \\
& \bar{Q}_{\mu}^{a}(z)=\partial^{v} \chi_{\nu \mu}^{a}(z)+Q_{\mu}^{a}(z) .
\end{aligned}
$$


Another functional differential identity can take care of (37) which reads,

$$
e^{\mathfrak{D}_{A}} \mathcal{F}_{1}[A] \mathcal{F}_{2}[A]=\left(e^{\mathfrak{D}_{A}} \mathcal{F}_{1}[A]\right) e^{\overleftrightarrow{\mathfrak{D}}}\left(e^{\mathfrak{D}_{A^{\prime}}} \mathcal{F}_{2}\left[A^{\prime}\right]\right)
$$

where the 'cross-linkage' operator $\exp \{\overleftrightarrow{D}\}$ is,

$$
\overleftrightarrow{\mathfrak{D}}=-i \int \stackrel{\overleftarrow{\delta}}{\frac{\delta}{\delta A}} D_{\mathrm{F}}^{(0)} \frac{\vec{\delta}}{\delta A^{\prime}}
$$

and the limit of $A_{\mu}^{a}=A_{\mu}^{\prime a}=0$ is to be taken in the end. Cumbersome, still standard calculations then give (up to an overall factor of $\exp \left(-\frac{1}{2} \operatorname{Tr} \ln \left(-D_{\mathrm{F}}^{(0)}\right)\right)$ to be included into the normalization),

$$
\begin{array}{cl}
e^{\mathfrak{D}_{A}} \mathcal{F}_{1}[A] \mathcal{F}_{2}[A]_{\left.\right|_{A=0}}=\quad & \mathcal{N} \exp \left[-\frac{i}{2} \int \bar{Q} \cdot \widehat{K}^{-1} \cdot \bar{Q}-\frac{1}{2} \operatorname{Tr} \ln \widehat{K}\right] \\
\cdot \exp \left[\frac{i}{2} \int \frac{\delta}{\delta A} \cdot \widehat{K}^{-1} \cdot \frac{\delta}{\delta A}-\int \bar{Q} \cdot \widehat{K}^{-1} \cdot \frac{\delta}{\delta A}\right] \\
\left.\cdot \exp (L[A])\right|_{A \rightarrow 0} \\
\bar{Q}_{\mu}^{a}(z)=\partial^{v} \chi_{\nu \mu}^{a}(z)+Q_{\mu}^{a}(z), \quad \widehat{K}_{\mu \nu}^{a b}=K_{\operatorname{spin}_{\mu \nu}}^{a b}+g f^{a b c} \chi_{\mu \nu}^{c} .
\end{array}
$$

Locality of the effective interaction and gauge independence again, whatever the initial gauge field function in use. However, it can be checked that the fundamental rule of duality, i.e., $g \rightarrow 1 / g$, is not verified contrarily to the pure YM case [6].

This is a non-trivial result, and the more so if auxiliary field integrations can be carried out in an exact way, as in the previous short version. However, so long as gauge invariance considerations are concerned only, this latter condition doesn't constitute any proviso. A figure can be proposed to illustrate the property of effective locality where it is important to point out that the pictured equality holds in a symbolic sense only [12].

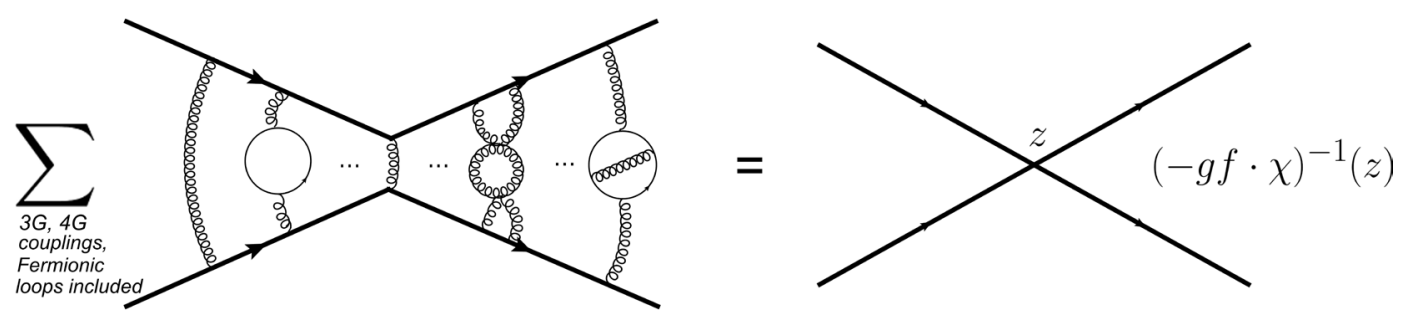

Figure 1. Effective locality: A symbolic pictorial representation

Now an unavoidable question is lurking about Fradkin's representations. Is effective locality dependent on these representations or is it more general a property? In order to catch the meaning of effective locality, it matters to answer this question. 


\subsection{Effective locality larger version}

The fermionic functional $L[A]$ of (5) is itself fully gauge invariant and local [11] so that the point can be examined in a quenched situation and in the simplest case of a 2-point Green's function. The relevant expression is thus,

$$
\begin{array}{r}
\mathcal{N} \int d[\chi] e^{\frac{i}{4} \int \chi^{2}} \\
\left.e^{\mathfrak{D}_{A}^{(o)}} e^{+\frac{i}{2} \int \chi \cdot F+\frac{i}{2} \int A \cdot\left(D_{F}^{(0)}\right)^{-1} A} G_{\mathrm{F}}(x, y \mid A)\right|_{A \rightarrow 0}
\end{array}
$$

with no particular representation being assumed for $G_{F}[A]$. This is,

$$
\left.\mathcal{N} \int d[\chi] e^{\frac{i}{4} \int \chi^{2}} e^{\mathfrak{D}_{A}^{(0)}} e^{+\frac{i}{2} \int A \cdot K \cdot A+i \int A_{a}^{\mu} \partial^{\nu} \chi_{\nu \mu}^{a}} G_{\mathrm{F}}(x, y \mid A)\right|_{A \rightarrow 0}
$$

with now,

$$
K=g f^{a b c} \chi_{\mu \nu}^{c}+\left.\left(D_{F}^{(0)}\right)^{-1}\right|_{\mu \nu} ^{a b} .
$$

Identifying the two functionals $\mathcal{F}_{1}[A]$ and $\mathcal{F}_{2}[A]$,

$$
\mathcal{F}_{1}[A]=\exp \left[\frac{i}{2} \int A \cdot K \cdot A+i \int A_{a}^{\mu} \partial^{v} \chi_{v \mu}^{a}\right], \quad \mathcal{F}_{2}[A]=G_{\mathrm{F}}(x, y \mid A)
$$

the functional differential identity (40) can be used again. Then, up to a global normalization factor of $\mathcal{N} e^{-\frac{1}{2} \operatorname{Tr} \ln \left(-D_{\mathrm{F}}^{(0)}\right)}$, the result can be written as,

$$
\begin{aligned}
e^{\mathfrak{D}_{A}} \mathcal{F}_{1}[A] \mathcal{F}_{2}[A]_{\left.\right|_{A=0}}=\quad & \exp \left[-\frac{i}{2} \int \nabla \chi \cdot(g f \cdot \chi)^{-1} \cdot \nabla \chi-\frac{1}{2} \operatorname{Tr} \ln (g f \cdot \chi)\right] \\
\cdot & \exp \left[\frac{i}{2} \int \frac{\delta}{\delta A} \cdot(g f \cdot \chi)^{-1} \cdot \frac{\delta}{\delta A}\right] \\
& \left.\cdot \exp \left[\int \nabla \chi \cdot(g f \cdot \chi)^{-1} \cdot \frac{\delta}{\delta A}\right] G_{\mathrm{F}}(x, y \mid A)\right|_{A \rightarrow 0} .
\end{aligned}
$$

The property of effective locality is manifest in the first line (47). In the second line (48) and third one (49), the functional $G_{\mathrm{F}}(x, y \mid A)$ isn't gauge invariant. But acted upon with the functional translation operator of (49) and the linkage operator of (48), the result becomes gauge invariant as soon as the prescription of $A \rightarrow 0$ is implemented.

The same applies trivially to a product of $n$ functionals, $\prod_{i=1}^{n} G_{\mathrm{F}}\left(x_{i}, y_{i} \mid A\right)$, that is to any $2 n$-point fermionic Green's function. The conclusion is that if Fradkin's representations must be introduced for the sake of actual calculations and derivations of further remarkable results [12], the gist of effective locality doesn't depend on them.

It may therefore be proposed that the gist of effective locality be the very way non abelian gauge invariance is realized in the non-perturbative regime of QCD.

Note that this proposition makes no restriction to the fermionic sector. This is because the pure bosonic sector (YM) admits a dual formulation (at least at leading order of a semi-classical expansion), and that this dual formulation is not only local, as it should, but also gauge-invariant (so long as the $\chi$ - field strength isn't gauged itself, as it isn't in the current fermionic case). 


\section{Conclusion}

The property of effective locality has been established on the basis of formal, yet standard functional manipulations and emerges as a non-perturbative property of QCD fermionic Green's functions, not bound to any approximation scheme artefact.

Effective locality has been able to display a number of promising tree-level consequences [1-5], while its meaning remained enigmatic. At variance with the pure Yang Mills euclidean case [6], in effect, this property cannot be thought of in terms of a dual form of the original QCD formulation.

Deriving effective locality, gauge-fixing appears mandatory in order to obtain well defined functional operations, but only as an intermediate step. This is made manifest by the trick of adding/subtracting a gauge-fixing density to the Lagrangian so that the original gauge-invariance is preserved from the onset and almost always manifest. As a consequence, the free-field gluonic functions one starts from, just disappear from the final Green's function calculations. This shouldn't be looked at as an odd statement because to calculate observables it is in no way mandatory to fix a gauge [13], even though an usage inherited from QED has long taken physicists to do so.

In the end the essential aspect of effective locality could very well be to reveal the very mode non-abelian gauge invariance is realized in the non-perturbative regime of QCD.

Contrarily to the perturbative regime of QCD where in full similarity to QED (though an order of magnitude more involved) gauge invariance is highly circuitous, the effective locality mode of gauge invariance realization is direct, free of ghost fields and of any Gribov's copies issues. This unexpected simplification could have some proportion or adequacy to the fact that it is in this non-perturbative sector only that real physical (hadronic) states show up in QCD, and nowhere else.

Further properties come about as one implement Fradkin's representations for the fermionic functionals, but these are the matter of an ongoing analysis [12].

\section{References}

[1] H.M. Fried, Y. Gabellini, T. Grandou, and Y.-M. Sheu, Eur. Phys. J. C65, 395 (2010).

[2] H.M. Fried, T. Grandou, and Y.-M. Sheu., Ann. Phys. 327, 2666 (2012).

[3] H.M. Fried, Y. Gabellini, T. Grandou, and Y.M. Sheu, Ann. Phys. 338, 107 ( 2013).

[4] H.M. Fried, T. Grandou, and Y.-M. Sheu, Ann. Phys. 344C, 78 (2014).

[5] H.M. Fried, P. H. Tsang, Y. Gabellini, T. Grandou, and Y.-M. Sheu, Ann. Phys. 359, 1 (2015).

[6] H. Reinhardt, K. Langfeld and L. v. Smekal, Phys. Lett. B, 300, 11 (1993); H. Reinhardt, "Dual description of QCD", arXiv:hep-th/9608191v1 (1996).

[7] H.M. Fried, T. Grandou, and R. Hofmann, Mod. Phys. Lett. A, Vol.32(2017) 1730030 (16 pages) (2017); arXiv:1706.02264v1 [hep-th].

[8] C. Itzykson and J.B. Zuber, Quantum Field Theory, ( McGraw-Hill Inc. 1980), p.448.

[9] M. B. Halpern, Phys. Rev. D16, 1798 (1977).

[10] T. Grandou, EPL 107, 11001 (2014). H.M. Fried, T. Grandou and R. Hofmann, Int. J. of Mod. Phys. A, 31, Nos. 20 \& 21, 1650120 (25 pages) (2016).

[11] H.M. Fried, Functional methods and eikonal models (Editions Frontières, Singapore, 1990), pp.85-87.

[12] H.M. Fried, T. Grandou, and R. Hofmann, work in progress.

[13] See A. Maas talk in XXVIII International Symposium on Lattice Field Theory, Lattice 2010, June 14-19 2010, Villasimius, Italy; arXiv.1010.5718v1 [hep-lat]. 